\title{
Development and content of a school-based occupational therapy intervention for promoting emotional wellbeing in children
}

\author{
Ema Tokolahi, PhD Candidate, Department of Rehabilitation and Occupation
}

Studies, Centre for Person-Centred Research, Auckland University of Technology, New Zealand.

Professor Clare Hocking, Department of Rehabilitation and Occupation Studies, Centre for Person-Centred Research, Auckland University of Technology, New Zealand.

Professor Paula Kersten, Department of Rehabilitation and Occupation Studies, Centre for Person-Centred Research, Auckland University of Technology, New Zealand.

Address correspondence to: Ema Tokolahi, MSc, Auckland University of Technology, Building AA, Private Bag 92006, Auckland 1142, New Zealand, etokolah@aut.ac.nz. 


\section{ABSTRACT}

The development and content of an occupational therapy intervention promoting emotional wellbeing, for children with subclinical anxiety, is described. Development and trialling followed a four-step process: (1) reviewing theory; (2) reviewing evidence; (3) incorporating expert opinion; (4) trialling. The intervention consists of eight group sessions, led by an occupational therapist, over 8 weeks of an academic term. It is proposed outcomes will be achieved by providing children with knowledge about health promoting occupations and how to participate in, balance and sustain these. This paper offers a guideline for the development and description of similar interventions to facilitate more robust evaluation of clinical practice.

\section{KEYWORDS}

Health promotion, mental health, occupational therapy, school-based, wellbeing 


\section{Introduction}

Anxiety disorders in childhood and adolescence are common, with studies from Australasia, Europe and North America indicating a period prevalence between 5\% and 31\% of whom only $10 \%$ fulfil criteria for a mental health disorder (Essau \& Gabbidon, 2013; Weare \& Nind, 2011). Anxiety symptoms include excessive levels of unhelpful internal cognitions that impact on the individual's participation in educational, social and family occupations and are associated with increased rates of anxiety and depression in early adulthood (Cresswell, Waite, \& Cooper, 2014). Subsequently, the burden of anxiety conditions on public health is significant, yet despite this many children remain untreated due to stigma associated with mental health interventions, lack of knowledge about mental health disorders and treatment options, and a reluctance to seek support (Yearwood \& DeLeon Siantz, 2010). This highlights the need for preventative and early interventions in this population, with schools offering a convenient and feasible location for the widespread delivery of preventative and targeted interventions to children (Essau \& Gabbidon, 2013; Stallard \& Buck, 2013).

For children and their families, occupational therapy may be viewed as a practical solution to impaired functioning associated with a child's symptoms of anxiety, without the stigma associated with traditional mental health interventions (Barney, Griffiths, Jorm, \& Christensen, 2006). Evaluation of occupational therapy as an intervention addressing mental health issues in children is notably absent in research literature. Additionally, systematic reviews of occupational therapy trials conducted in different settings or with different populations often report interventions that are inadequately described, limiting the possibility of synthesizing results, replicating studies or generalizing findings to practice (Campbell et al., 2000). This paper aims to describe the development and content of an occupational therapy intervention that is currently being evaluated in a cluster randomized controlled trial. 
Details of the trial protocol are described elsewhere (Tokolahi, Hocking, Kersten, \& Vandal, 2014).

\section{Defining constructs}

The broad conceptualization of what constitutes mental health and the client-centred and complex nature of occupational therapy contributes to difficulties researchers have in defining and evaluating such interventions. For the purpose of this paper, mental health is defined as a state of emotional, social and psychological wellbeing; the individual is productive; is able to adapt to changes or adversity; is able to maintain positive social relationships; and has positive self-esteem (Weare \& Nind, 2011; Yearwood \& DeLeon Siantz, 2010). Occupational therapy focuses on 'the nature, balance, pattern and context of occupations and activities in the lives of individuals, family groups and communities' (Creek, 2003, p. 8). The main aim of therapy is to enable the individual to make occupational choices that maintain, restore and provide benefit in the context of their individual needs, the demands of their daily activities and their environment (Creek, 2003).

\section{Methods}

Development of occupational therapy interventions across a range of settings typically follows a process of reviewing evidence, consulting with experts and piloting the protocol (Sackley, Atkinson, \& Walker, 2004). Two studies outlined the development of an intervention manual (Cook \& Birrell, 2007; Maris \& Bradshaw, 2004) and another the development of a framework for practice alongside a suggested frequency, duration and 'dose' of occupational therapy (Sackley et al., 2004); both methods facilitating intervention replication in different contexts. A common theme described by these authors was the challenge of defining occupational therapy interventions with sufficient flexibility to enable 
therapists to take a holistic, client-centred approach; however, it was asserted that 'it is possible to define a specific intervention in a clearly defined group' (Sackley et al., 2004, p. 104). This paper focuses on the development and content of Kia Piki te Hauora: Uplifting our Health and Wellbeing - a school-based group intervention for children with subclinical levels of anxiety - using a four-step process similar to that used by Sackley et al. (2004): (1) reviewing the theory; (2) reviewing the evidence; (3) incorporating expert opinion; and (4) trialling.

\section{Step 1: Reviewing the theory}

Middle childhood (defined in this study as 10-14 years) is a period of substantial physical, emotional and social changes. Cognitive changes mean children have the ability for more complex thought, are more able to verbalize their experiences and emotions and are developing their own sense of right and wrong. Evidence suggests that middle childhood is a key time for targeting preventative interventions (Dadds, Spence, Holland, Barrett, \& Laurens, 1997; Horowitz, Garber, Ciesla, Young, \& Mufson, 2007), in part because research across the lifespan has established that self-esteem becomes more stable in adolescence (defined in this study as 12-17 years) and early adulthood (Trzesniewski, Donnellan, \& Robins, 2003). That finding further supports early adolescence as a key time in a child's development for interventions aimed at promoting self-esteem before it stabilizes.

Engaging in a balance of occupations is vital for children to achieve optimal development and experience health and wellbeing (Bazyk \& Bazyk, 2009). The range of occupations they participate in, the meaning they attribute to those occupations and the level or intensity with which they chose to, need to, or are expected to participate in various occupations will all change as they progress through each developmental stage (Davis \& Polatajko, 2014). There is a dynamic, reciprocal interaction between the occupation and the person so that 
participation in an occupation influences, and is influenced by, individual's beliefs, identities, experiences, goals and life narratives; concepts that are still very much emerging in children. The Dynamic Performance Analysis framework for occupational performance assumes healthy occupational performance depends on three, hierarchical levels: knowledge of how to perform; motivation to perform; and the ability to perform (Polatajko, Mandich, \& Martini, 2000). However, children, more so than adults, often lack the knowledge or skills to determine the health-relevant consequences of the occupations they chose to participate in (Jackson, Carlson, Mandel, Zemke, \& Clark, 1998). As a result, there is an identified need to develop an intervention aimed at educating and encouraging children to 'construct daily routines in a manner that would optimize their health and psychosocial wellbeing' and enabling them to perform these occupations in real-life environments (Jackson et al., 1998, p. 329).

Use of occupations as transformative tools to promote mental health and wellbeing therefore has the potential to be universally meaningful and help shape children's decisionmaking to support better mental health outcomes. This approach was clearly documented in the Well Elderly Program and described as ‘Lifestyle Redesign’ (Mandel, Jackson, Zemke, Nelson, \& Clark, 1999). Given the developmental stage and emerging self-awareness in the population this intervention is targeting - middle childhood - it would be more appropriate to consider this intervention 'Lifestyle Design'. Developmentally appropriate occupations for children may include games, group discussions, brainstorming, and paper-pencil tasks and should be accompanied by opportunities to engage in occupational analysis or reflection to understand the reciprocal relationships between doing, thinking and feeling (Bazyk \& Bazyk, 2009; Jackson et al., 1998).

An ecological focus highlights the importance of considering the developing child as a whole and on 'strengthening relationships in the life space of a child's family, school, peers 
and community’ (Brendtro \& Mitchell, 2011, p. 8). Schools play an important role in children’s social and educational development (Stallard \& Buck, 2013) and can provide an avenue for therapists to work with children who may not otherwise access therapeutic interventions (Arbesman, Bazyk, \& Nochajski, 2013; Stallard \& Buck, 2013; Weare \& Nind, 2011).

\section{Step 2: Reviewing the evidence}

We reviewed experimental research that explored the range of preventative mental health interventions available for children in the school environment. The tiers of public health promotion in this environment could be understood as:

- Tier 1: Universal or whole-school approach; interventions provided to all children at a school regardless of symptom presence or absence; aimed at competence enhancement.

- Tier 2: Targeted or indicated interventions; designed to support smaller groups of children from a school who are at risk of developing mental health challenges with an increasing focus on enhancing protective factors as well.

- Tier 3: Intensive interventions that are individually-focused; aimed at diminishing the effects of identified mental, emotional or behavioral disorders in specific children (Arbesman et al., 2013).

Our review found that universal interventions are typically not universally effective, with greater benefits being measured in those with greater needs at baseline (Dadds et al., 1997; Horowitz et al., 2007). Additionally, the majority of interventions described demonstrated the effectiveness of Cognitive Behavior Therapy (CBT) at modifying an individual's internal cognitions to reduce symptoms of anxiety in children (Cresswell et al., 2014; Dadds et al., 1997; Horowitz et al., 2007). However, CBT has been found insufficient in effecting 
significant change when level of functioning was measured as an outcome in children (Vitiello et al., 2006).

A substantial systematic review of 52 systematic reviews and meta-analyses, by Weare and Nind (2011), identified the following key evidence-based principles for effective interventions aimed at mental health promotion and prevention in schools that were considered in the development of Kia Piki te Hauora: Uplifting our Health and Wellbeing:

- targeted interventions are generally demonstrated to be more effective than universal interventions, perhaps due to a 'ceiling effect' for those without problems and for whom there is less scope for change;

- developing skills and competence is a central part of any comprehensive and effective intervention;

- a combination of behavioral (e.g. games, simulations, group work) and didactic strategies is associated with better outcomes than either approach alone;

- most interventions require specialist clinical staff to develop and disseminate the intervention initially, however, to become sustainable and embedded in school culture other school staff (e.g. teachers) need to be able to take over;

- family involvement supports generalizing of skills outside the intervention context;

- there is evidence to support short term interventions (8-10 weeks) targeting specific or mild concerns with longer durations (9 months to a year) for more severe problems;

- interventions result in better outcomes when they are of a high quality i.e. developed on sound theoretical knowledge, administered by trained leaders, explicit focus on the desired outcome (rather than using a different focus and hoping for indirect effects), manualized or with clear guidelines and implemented completely and accurately. 
Traditionally, positive outcomes have focused on the mitigation of mental health problems (such as symptoms of anxiety or depression), however, there is increasing focus on building protective factors (such as self-esteem, participation and wellbeing) as a legitimate, if not more effective, method for promoting mental wellbeing and preventing the onset of disease. Yearwood and DeLeon Siantz (2010, p. 513) reported 'positive mental health is a powerful protective factor against mental illness. In the United Kingdom, a review of the most up-to-date evidence for strategies aimed at improving wellbeing in everyday life identified a set of five, simple actions that focus on building protective factors: connection with others; giving; noticing; continued learning; and getting active (The New Economics Foundation, 2011). These principles and actions were incorporated into the design and content of Kia Piki te Hauora: Uplifting our Health and Wellbeing.

Occupational therapists, in particular, can offer important contributions for addressing childhood anxiety to all three tiers of service in the public health model, including, amongst others: reducing risk factors and symptoms through engagement in occupation; providing skill development training in the context of everyday occupations; and providing training in adaptation to change and coping with adversity to promote mental health (Arbesman et al., 2013). An example of preventative occupational therapy with a younger population is the Occupational Therapy Groups for HOPE (Healthy Occupations for Positive Emotions) for low-income urban youth (Bazyk \& Bazyk, 2009).

Arbesman and Bazyk (Arbesman et al., 2013) conducted a systematic review of mental health promotion, prevention and intervention literature for children and youth, which largely described occupation- or activity-based interventions published by researchers outside the field of occupational therapy, that fitted within the scope of occupational therapy practice. Themes identified were: social skills programming; health promotion e.g. stress management, managing obesity, health literacy; and leisure, recreation and play activities. The authors 
concluded there was good evidence to support 'an occupation- and activity-based approach that can be used with children and youth at all three tiers in a wide range of environments' (Arbesman et al., 2013, p. 125). Consistent with these themes Bazyk and Bazyk (2009) also identified enjoyment and skill development as important for children and youth to engage with interventions.

In summary, while the discipline-specific evidence for preventative occupational therapy interventions with children is sparse, there is sound evidence for the components of such interventions, and for preventative occupational therapy in other contexts.

\section{Step 3: Incorporating expert opinion}

An indicated, preventative-occupational therapy group intervention protocol was developed based on the reviewed theory and evidence and the first author's clinical experience of running groups in the context of mental health clinics for children with anxiety (Tokolahi, Em-Chhour, Barkwill, \& Stanley, 2013). To ensure the intervention was appropriate in a school setting, consultation was carried out with the following groups:

- Children eligible to participate in the intervention: age range 11-13 years $(n=18)$.

- Senior teaching staff at eligible schools, such as Principals and/or Special Education Needs Coordinators (SENCOs) $(n=4)$.

- Cultural Advisors with knowledge of providing culturally sensitive and safe interventions, with a strong mental health component, to children $(n=3)$.

- Occupational therapists with knowledge and experience of providing and teaching occupational therapy, with prior experience in working with children and/or in a mental health context $(n=7)$. 
Consultation with children occurred during the trialling phase described in step 3. SENCOs from four different schools were consulted in the planning of the intervention logistics and overall content. All had a minimum 6 years of experience teaching. Their advice about the amount of time schools would be willing to release children from the standard curriculum during the week to facilitate their attendance at a wellbeing group varied (60-120 minutes) and so the duration feasible across them all was selected to ensure consistency i.e. 60 minutes. Including parents in the intervention through face-to-face group sessions was considered, given evidence that involving them can significantly increase an intervention’s effectiveness (Arbesman et al., 2013; Khanna \& Kendall, 2009). The SENCO's consulted expressed concern that while a parenting component could be helpful it risked stigmatising the children involved and raised significant practical complications. Direct parent involvement is therefore not directly included in Kia Piki te Hauora: Uplifting our Health and Wellbeing; however, parents consent to their child's participation and are informed their children will be given skills to practice between sessions so they can be involved with these if they choose. Feedback on the intervention protocol from the SENCOs was positive and they suggested additional components to keep the program consistent with the national curriculum, such as safe participation in cyber occupations.

This intervention was developed in the context of New Zealand, a bicultural nation, in which demonstrating the cultural sensitivity of interventions is paramount (Putaiora Writing Group, 2012). While the intervention does not target ethnic groups specifically, it is anticipated people with a variety ethnicities will be involved e.g. children, parents/caregivers or school personnel. Three cultural advisors of Māori and Pacific descent were therefore consulted about the design, look and implementation of the intervention protocol; all had a minimum 10 years of experience working in mental health services. After the discussions about the content and implementation strategies with the advisors, time was allowed for them 
to consult with peers before again meeting with the researcher to validate or adjust their feedback. Modifications made included the gifting of the intervention name, Kia Piki te Hauora (Māori, translated into English means Uplifting our Health and Wellbeing) and the adaptation of an introductory cultural protocol to incorporate an occupational focus.

An expert panel of occupational therapists all had a minimum of eight years clinical experience. The panellists were asked if they recognized the intervention as being occupational therapy and what modifications or additions would they recommend. All agreed the intervention was clearly recognizable as occupational therapy, suggesting content validity, and recommended a number of modifications to 'de-clutter' the sessions and improve content clarity and focus. This included the creation of posters outlining key messages and objectives for each session; the addition and removal of some activities (e.g. added "blowing bubbles” as a breathing activity; removed "Defining problem solving” as an activity); capitalising on the goal setting required for skills practice within and between sessions rather than focusing on this as a separate topic; modifying hand-outs to support those with handwriting difficulties; and more consistent terminology.

\section{Step 4: Trialling}

The modified version of the intervention protocol was then evaluated by the first author by trialling it in two different schools, to inform its further development and test how the group format would work in a school environment. The trial was organised through a private occupational therapy practice, but offered on a no cost basis as its effectiveness had not been proven. Eighteen children aged 10-12 years participated in the two trials. Overall feedback from the children was positive - they enjoyed the intervention, in particular the activities, and felt they had learned something in the process. Minor modifications were made following their feedback and observations of how they participated in the intervention. Modifications 
included the removal of explicit didactic teaching about goal setting as this was already incorporated into several direct experience activities; substituted one of the relaxation activities to convey the same technique in a more developmental-appropriate manner; and removing some games that were less obviously associated with each session's key messages (e.g. a game identifying sugar content in drinks typically available on the way home from school). Overall, this resulted in a better paced and more cohesive intervention protocol. The SENCO from one of the schools reported the positive feedback she received from children and parents was sufficient for her to consider the intervention successful and expressed interest in offering the intervention at her school in the future.

\section{Results}

Figure 1 depicts the refined Kia Piki te Hauora: Uplifting our Health and Wellbeing intervention model, including its methods of delivery, session topics, proposed dynamic changes in occupation and the subsequent health related outcomes anticipated. Place Figure

\section{1 here}

\section{Methods of intervention delivery}

Kia Piki te Hauora: Uplifting our Health and Wellbeing has been manualized based on the protocol developed and described in this article. The intervention is delivered by an occupational therapist with a group of 7-12 children from Years 7 and 8 (ages 11-13 years): this is a discrete age bracket in the New Zealand educational system known as 'intermediate'. The intervention runs for 1 hour a week over a period of 8 weeks of a school term. Each 1 hour session follows a similar format: warm-up activity, skill development, skill practice, discussion about application of skill to real-life, closing round and skills practice for between sessions. The methods of intervention delivery mirror those described for the Well Elderly 
Program (Mandel et al., 1999): didactic presentation, peer exchange, direct experience and personal exploration. All four delivery modes are integrated into every session to encourage a dynamic and interactive process. Didactic activities include increasing the repertoire of terms, concepts and experiences children are able to name and describe; information sharing e.g. national recommendations regarding duration of hours of sleep or daily physical activity; and skill development for overcoming difficult emotions, such as experiencing different relaxation techniques. Peer exchange is explicitly and implicitly woven into activities to encourage normalisation of experiences, brainstorm ideas and encourage effective positive and informative communication. Direct experience involves children actively participating in a range of developmentally-appropriate experiences to highlight concepts and practice skills for managing strong emotions e.g. relaxation, games and planning. During personal exploration the children engage in occupational self-analysis, pepeha ${ }^{1}$, and activity scheduling to promote understanding of how their occupational choices impact on their health, self-esteem, participation and wellbeing. Children are encouraged to plan, build and design healthy routines, behaviors and habits in their day-to-day life.

\section{Session topics}

Introduction to occupation: Children are introduced to each other and the occupational terminology and language used throughout the program. Through developmentally appropriate play, terms such as ‘occupations', ‘hidden occupations', ‘main occupations’ and 'wellbeing' are discussed to ensure they have a reasonable grasp of these concepts.

Sleep and rest occupations: After reflecting on their own balance of rest or sleep occupations in relation to other occupations, children are given information about and encouraged to reflect on why these occupations are important, how occupational choices

\footnotetext{
1 'Pepeha' refers to a way of introducing one’s self in Māori: in this context the pepeha has been modified to have an occupational focus and was developed in consultation with Māori cultural advisors.
} 
made during the day can impact on their participation in sleep or rest occupations, and how their own occupational choices compare with national recommendations for their age-group.

Active occupations: Children reflect on their own balance of active occupations in relation to other occupations and they are encouraged to consider why these occupations are important, how occupational choices made during the day can inhibit or promote healthy physical activity and how their own occupational choices compare with national recommendations for their age-group.

Communication in occupations: Games are used to illustrate the differences between verbal, non-verbal and cyber communication, the role and impact of each of these and ideas shared about how occupations can depend on and be enhanced by effective communication.

Occupational disruption: Each child is asked to reflect on how their participation in occupations has been affected by emotional distress and the subsequent impact this had on further occupational engagement e.g. bullying, exams, mental ill-health. Underlying physical, cognitive and affective components that contribute to such disruption are discussed.

Coping occupations: A range of occupation-based strategies for overcoming difficult emotions, which could restore or promote occupational participation, are practiced and discussed. These include sensory strategies, having an action plan and relaxation.

Values and identity occupations: This is very much an area of development in this population. Children are encouraged to explore what is important to them personally and how they wish to be perceived by others, then compare this with the occupational choices they make on a daily basis.

Integrative summary: A review of the previous topics is undertaken to consolidate learning and experiences. Further, children are actively encouraged to reflect on their personal gains from participating in the group. The program concludes with a celebratory 
ceremony in which children are presented with positive feedback from peers, the program manual (that contains the content and worksheets from previous sessions) and a certificate of attendance.

\section{Proposed dynamic changes in occupation and subsequent health related outcomes}

Children participating in this intervention are supported to progress through the framework of the Dynamic Performance Analysis hierarchy in relation to their approach to occupations and occupational choices generally, rather than with regard to a specific occupation. It is proposed this is achieved by providing children with knowledge about what constitutes a health promoting occupation and its benefits, resulting in motivation to participate in health promoting occupations and information about how to participate in, balance and sustain these. Consequently, it is hypothesized the subsequent outcomes are a reduction in risk factors (symptoms of anxiety and depression) and an increase in protective factors (selfesteem, participation and wellbeing).

\section{Discussion and conclusion}

\section{Discussion}

It is important for children and their families to have access to evidence-based interventions that promote the child's health and wellbeing and reduce the likelihood of developing mental health problems in the future (Arbesman et al., 2013). Identifying interventions that reduce the lifetime impact of mental health issues is an important focus of government policies internationally (DH/Health Improvement Directorate, 2005; Mental Health Commission, 2012; US Department of Health and Human Services, 1990). Research into the effectiveness of occupational therapy interventions is a top research priority for the profession and in order 
to achieve this careful and accurate description of interventions is required (College of Occupational Therapists, 2007; The American Occupational Therapy Foundation, 2013).

\section{Conclusion}

This paper has described the development and given an overview of Kia Piki te Hauora: Uplifting our Health and Wellbeing, a manualized, indicated occupational therapy group intervention. It uses engagement in developmentally appropriate activities to promote mental health and well-being by enabling students to understand the relationship between what they do and how this influences their identity, self-concept, health and wellbeing; to practice and develop strategies for overcoming difficult emotions; and to apply this knowledge in designing and building healthy routines, behaviors and habits in their day-to-day life. This intervention is currently being evaluated in a cluster randomized controlled trial (Tokolahi et al., 2014).

\section{Practice implications}

The intervention described has been developed from theory and evidence-based practice; it was reported as acceptable and to have content validity as determined by the stakeholders consulted. Thus, if the cluster randomized controlled trial (in progress (Tokolahi et al., 2014)) demonstrates sufficient effectiveness of this intervention for promoting wellbeing in children, there is scope for introducing it into the health promotion component of schools' curricula. The process described in this paper could be used by other practitioners when developing interventions or seeking to define current interventions more accurately to facilitate more robust evaluation of clinical practice. 


\section{Acknowledgements}

We would like to thank those involved in the consultation process of the intervention design:

children and school personnel who participated in early trials; cultural advisors; and the occupational therapy expert panel: Linda Bowden, Mathijs Luccasen, Ellen Nicholson, Suzanne Patterson and others who prefer to remain unnamed. 


\section{References}

Arbesman, M., Bazyk, S., \& Nochajski, S. M. (2013). Systematic review of occupational therapy and mental health promotion, prevention, and intervention for children and youth. American Journal of Occupational Therapy, 67(6), e120-130. doi:10.5014/ajot.2013.008359

Barney, L. J., Griffiths, K. M., Jorm, A. F., \& Christensen, H. (2006). Stigma about depression and its impact on help-seeking intentions. Australian and New Zealand Journal of Psychiatry, 40(1), 51-54. doi:10.1080/j.1440-1614.2006.01741.x

Bazyk, S., \& Bazyk, J. (2009). Meaning of occupation-based groups for low-income urban youths attending after-school care. American Journal of Occupational Therapy, 63(1), 69-80. doi:10.5014/ajot.63.1.69

Brendtro, L. K., \& Mitchell, M. L. (2011). Practice-based evidence: Families as life span experts. Reclaiming Children and Youth, 20(3), 8-14.

Campbell, M., Fitzpatrick, R., Haines, A., Kinmouth, A. L., Sandercock, P., Spiegelhalter, D., \& Tyler, P. (2000). Framework for design and evaluation of complex interventions to improve health. British Medical Journal (Clinical Research Ed.), 321(7262), 694-696. doi:10.1136/bmj.321.7262.694

College of Occupational Therapists. (2007). Building the evidence for occupational therapy: Priorities for research. Retrieved 14 April, 2013, from http://www.cot.co.uk/uk-otresearch-foundation-ukotrf/ukotrf-research-priorities

Cook, S., \& Birrell, M. (2007). Defining an occupational therapy intervention for people with psychosis. British Journal of Occupational Therapy, 70(3), 96-106.

Creek, J. (2003). Occupational therapy defined as a complex intervention. London: College of Occupational Therapists.

Cresswell, C., Waite, P., \& Cooper, P. J. (2014). Assessment and management of anxiety disorders in children and adolescents. Archives of Disease in Childhood, 99(7), 674678. doi:10.1136/archdischild-2013-303768

Dadds, M. R., Spence, S. H., Holland, D., Barrett, P. M., \& Laurens, K. R. (1997). Prevention and early intervention for anxiety disorders: A controlled trial. Journal of Consulting and Clinical Psychology, 65(4), 627-635. doi:10.1037/0022-006X.65.4.627

Davis, J., \& Polatajko, H. (2014). Occupational development. In C. H. Christiansen \& E. A. Townsend (Eds.), An introduction to occupation: The art of science and living (2nd ed., pp. 113-152). Essex, UK: Pearson.

DH/Health Improvement Directorate. (2005). Shaping the future of public health: Promoting health in the NHS. London: Author.

Essau, C. A., \& Gabbidon, J. (2013). Epidemiology, comorbidity and mental health service utilization. In C. A. Essau \& T. H. Ollendick (Eds.), The Wiley-Blackwell handbook of the treatment of childhood and adolescent anxiety (pp. 23-42). Chichester, UK: WileyBlackwell.

Horowitz, J. L., Garber, J., Ciesla, J. A., Young, J. F., \& Mufson, L. (2007). Prevention of depressive symptoms in adolescents: A randomized trial of cognitive-behavioral and 
interpersonal prevention programs. Journal of Consulting and Clinical Psychology, 75(5), 693-706. doi:10.1037/0022-006X.75.5.693

Jackson, J., Carlson, M., Mandel, D., Zemke, R., \& Clark, F. (1998). Occupation in lifestyle redesign: The well elderly study occupational therapy program. American Journal of Occupational Therapy, 52(5), 326-336. doi:10.5014/ajot.52.5.326

Khanna, M. S., \& Kendall, P. C. (2009). Exploring the role of parent training in the treatment of childhood anxiety. Journal of Consulting and Clinical Psychology, 77(5), 981-986. doi:10.1037/a0016920

Mandel, D., Jackson, J., Zemke, R., Nelson, L., \& Clark, F. (1999). Lifestyle redesign: Implementing the Well-Elderly Program. Bethesda, MA: AOTA.

Maris, H., \& Bradshaw, T. (2004). Life skills training in schizophrenia. British Journal of Occupational Therapy, 67(5), 217-224.

Mental Health Commission. (2012). Blueprint II: Improving mental health and wellbeing for New Zealanders. Wellington, NZ: Ministry of Health.

Polatajko, H., Mandich, A., \& Martini, R. (2000). Dynamic performance analysis: A framework for understanding occupational performance. American Journal of Occupational Therapy, 54(1), 65-72. doi:10.5014/ajot.54.1.65

Putaiora Writing Group. (2012). Te ara tika: Guidelines for Maori research ethics: A framework for researchers and ethics committee members. Wellington, NZ: Health Research Council of New Zealand.

Sackley, C. M., Atkinson, J. C., \& Walker, M. F. (2004). Occupational therapy in nursing and residential care settings: A description of a randomised controlled trial intervention. British Journal of Occupational Therapy, 67(3), 104-110.

Stallard, P., \& Buck, R. (2013). Preventing depression and promoting resilience: Feasibility study of a school-based cognitive-behavioural intervention. British Journal of Psychiatry: Supplement, 54, s18-23. doi:10.1192/bjp.bp.112.119172

The American Occupational Therapy Foundation. (2013). Research Priorities and Parameters of Practice for Occupational Therapy. Retrieved 14 April, 2013, from http://www.aotf.org/resourceswlwlibrary/researchprioritiesandparametersofpractice. $\underline{\operatorname{aspx}}$

The New Economics Foundation. (2011). Five ways to wellbeing: New applications, new ways of thinking. London, UK: Author.

Tokolahi, E., Em-Chhour, C., Barkwill, L., \& Stanley, S. (2013). An occupation-based group for children with anxiety. British Journal of Occupational Therapy, 76(1), 31-36. doi:10.4276/030802213X13576469254694

Tokolahi, E., Hocking, C., Kersten, P., \& Vandal, A. C. (2014). Cluster-randomised controlled trial of an occupational therapy group intervention for children designed to promote emotional wellbeing: Study protocol. BMC Psychology, 2(16). doi:10.1186/2050-72832-16 
Trzesniewski, K. H., Donnellan, M. B., \& Robins, R. W. (2003). Stability of self-esteem across the life span. Journal of Personality and Social Psychology, 84(1), 205-220. doi:10.1037/0022-3514.84.1.205

US Department of Health and Human Services. (1990). Healthy people 2000: National health promotion and disease prevention objectives. Washington, DC: Author.

Vitiello, B., Rohde, P., Silva, S., Wells, K., Casat, C., Waslick, B., ... Team, T. (2006). Functioning and quality of life in the Treatment for Adolescents with Depression Study (TADS). Journal of the American Academy of Child and Adolescent Psychiatry, 45(12), 1419-1426. doi:10.1097/01.chi.0000242229.52646.6e

Weare, K., \& Nind, M. (2011). Mental health promotion and problem prevention in schools: What does the evidence say? Health Promotion International, 26 Supplement 1, i2969. doi:10.1093/heapro/dar075

Yearwood, E. L., \& DeLeon Siantz, M. L. (2010). Global issues in mental health across the life span: Challenges and nursing opportunities. Nursing Clinics of North America, 45(4), 501-519. doi:10.1016/j.cnur.2010.06.004 